\title{
Philosophiques
}

\section{L’auto-méditation phénoménologique pour une communauté des philosophes}

\section{Rémi Tremblay}

Volume 7, numéro 1, avril 1980

URI : https://id.erudit.org/iderudit/203129ar

DOI : https://doi.org/10.7202/203129ar

Aller au sommaire du numéro

Éditeur(s)

Société de philosophie du Québec

ISSN

0316-2923 (imprimé)

1492-1391 (numérique)

Découvrir la revue

Citer cet article

Tremblay, R. (1980). L’auto-méditation phénoménologique pour une communauté des philosophes. Philosophiques, 7(1), 3-39.

https://doi.org/10.7202/203129ar d'utilisation que vous pouvez consulter en ligne.

https://apropos.erudit.org/fr/usagers/politique-dutilisation/ 


\title{
L'AUTO-MÉDITATION PHÉNOMÉNOLOGIQUE POUR UNE COMMUNAUTÉ DES PHILOSOPHES*
}

\author{
par Rémi Tremblay
}

\begin{abstract}
Dans la conclusion de La crise des sciences européennes et la phénoménologie transcendantale, Husserl invite les lecteurs à effectuer l'opération phénoménologique qui permettrait de retrouver le sens de la Raison à l'œuvre dans l'histoire, mais qui s'est perdu au cours de la "crise " actuelle de l'humanité européenne. Cette opération s'appelle "l'auto-méditation", Selbstbesinnung. La présente étude cherche à définir l'auto-méditation, à montrer dans quel contexte Husserl l'introduit, quels sont ses principaux caractères et les buts qu'elle vise. Elle cherche également à savoir si l'auto-méditation, par son processus même, peut vraiment résoudre les problèmes qui ont produit cette crise de la "perte du sens ».
\end{abstract}

Notre étude s'insère dans la démarche de certaines critiques à l'endroit de l'idéalisme husserlien, entre autres celle de Habermas qui doute que Husserl ait "le droit de revendiquer pour la phénoménologie en tant que théorie pure quelque efficacité au niveau de la pratiquel", car elle demeure, même en se présentant comme une "auto-réflexion "(Selbstreflexion), une théorie au sens traditionnel qui "dissocie définitivement la connaissance de l'intérêt ${ }^{2}$ ". Habermas reproche à Husserl de retomber « dans une autre forme d'objectivisme ${ }^{3}$ ", dont il voulait pourtant se libérer. Il ne voit donc pas dans la méthode phénoménologique le moyen

* Je tiens à temercier M. J. Danek, qui m'a conseillé tout au long de l'étude de certe thémarique, ainsi que $\mathrm{M}$. G. Bouchard, qui m’a suggéré plusieurs corrections pour améliorer la présentation de ce texte.

1. Jürgen Habermas, La teshnique et la science camme idéslngie, trad. par J.R. Ladmiral, Paris, Les Essais, Gallimard, 1973, p. 141.

2. Ibid., p. 140

3. Ibid, p. 142 
permettant à l'humanité de "s'émanciper " véritablement. En nous attachant au texte de la Krisis . . ., nous nous demanderons si l'auto-méditation, en tant qu'elle cherche à renouer avec l'idéal de la philosophie-guide-de-l'humanité, est apte à produire une émancipation de l'humanité.

\section{Les symptômes de la crise et son remède : l'auto-méditation}

De façon générale, l'état de crise vécu par l'humanité européenne est ce qui a motivé l'idée d'une auto-méditation. Dans la Krisis . . .4. Husserl la présente explicitement comme une méthode permettant de surmonter l'impasse créée par la crise des sciences et de la philosophie ${ }^{5}$, ou encore comme une voie décisive permettant à l'humanité de se comprendre et de diriger son destin de façon rationnelle ${ }^{6}$.

Cette crise se manifeste principalement par des symptômes au niveau des sciences qui ont perdu leur « importance pour la vie " en excluant «les questions qui portent sur le sens ou sur l'absence de sens de toute cette existence humaine " $(\S 2, \text { p. } 10)^{7}$. Les sciences positives, devenues des techniques, se sont de plus en plus éloignées de l'idéal d'une science universelle, élaboré à la Renaissance, qui voulait créer une forme "philosophique " d'existence ( $\$ 3$, p. 12), c'est-à-dire donner une forme de raison à l'existence humaine et au monde qui l'entoure. L'écart se creusant d'une façon de plus en plus marquée entre le développement des sciences et l'idéal d'une science philosophique, on assiste alors à "l'effondrement de la foi " dans les possibilités de la méthode philosophique pour

4. Edmund Husserl, Di Krisis der europäischen Wissenschaften und die Transzendentale Pbänomenologie, Herausgegeben von W. Biemel, Den Haag, Martinus Nijhoff, 1962.

5. "Il [Husserl] eut de prime abord la conviction que la méthode phénoménologique constituait le moyen de susciter chez les hommes la transformation d'atritude et de compréhension de soi, par laquelle cette situation de crise pourrait être surmontée. " Ludwig Landgrebe, "La signification de la phénoménologie de Husserl pour la réflexion de notre époque"; version abrégée d'une conférence traduite par J. Taminiaux, dans Husserl et la pensée moderne, Actes du $2^{e}$ colloque international de phénoménologie, 1-3 nov. 1956 ; La Haye, Martinus Nijhoff, 1959, p. 225.

6. D'autres textes husserliens développent une semblable façon de voir à propos de l'auto-méditation, telles les leçons 28,29,30, 52 de Pbilosopbie Première 2, ainsi que l'" Introduction " et le paragraphe 104 de Logique formelle et Logique transcendantale.

7. Edmund Husserl, La crise des sciences européennes et la phénoménologie transcendantale, trad. $G$. Granel, Paris, Gallimard, 1976, § 2, p. 10. Nous utilisons ce systeme d'abréviation pour toutes les citations qui réferent à la Krisis . . . dans sa traduction française. 
guider l'existence humaine ( $\$ 5$, p. 18$)$. On se satisfait des succès pratiques obtenus par les sciences positives dans leurs applications techniques, mais on perd de vue leur fondement philosophique. Cependant, malgré ces succès apparents, Husserl constate tout de même une crise existentielle au cour de l'humanité européenne, qui révèle qu'elle est gravement «malade » (Annexe III, p. 338).

Devant ces symptômes alarmants, il propose donc un remède, une "médecine des nations " afin que cette humanité retrouve le sens rationnel qui lui fait défaut. Ce remède consiste dans l'adoption de l'attitude transcendantale et de la méthode phénoménologique, plus précisément dans l'exercice d'une automéditation radicale. Selon Husserl, la crise, elle-même, survient peut-être du fait que les hommes n'ont pas entrepris ou ont mal compris « la tâche d'auto-méditation que la situation d'« effondrement» de notre époque, y compris «l'effondrement de la science », a fait naître pour nous » ( $\$$ I, p. 68). C'est ainsi que le savant, en n'exerçant pas en lui un "questionnement-en-retour ${ }^{8}$ adéquat, en est venu à se détourner du sens original et effectif de la méthode scientifique ( $\$ 9 \mathrm{k}$, p. 65-66) et, par le fait même, du sens originel qu'elle avait pour l'existence humaine. De façon générale, "l'insatisfaction » et la déception, causées par l'incapacité de la science et de la philosophie à donner un sens rationnel à la vie humaine, motivent l'idée d'une auto-méditation afin de comprendre et de régler les causes de cette insatisfaction.

Mais outre la « situation d'effondrement » ou l' « insatisfaction » qui inspire l'idée d'une auto-méditation, l'existence même du philosophe, en tant qu'il se décide pour la philosophie, exige de sa part une méditation sur ce qui fonde sa tâche. L'existence philosophique débute avec ce type de méditation. À ce propos, Husserl se réfere constamment au philosophe-débutant exemplaire, Descartes, qui pose la nécessité de la méditation radicale pour celui qui veut répondre d'une manière absolue de la validité des connaissances, donc qui veut philosopher (App. X, p. 469) ${ }^{9}$. Le retour au sens

8. Questionner-en-retour est un processus inhérent à l'auto-méditation.

9. Cette idée est déjà élahorée dans les leçons 28 et 29 de Philosophie Première 2, et dans l'"Introduction" des Méditations Cartésiennes. 
originel et son accomplissement authentique passent par l'auto-méditation. Cependant, qu'est-ce qui définit précisément cette auto-méditation? Qu'est-ce qui la caractérise essentiellement, quels sont ses buts et comment fonctionne-telle? Décrivons d'abord ses principaux caractères :

\section{Principaux caractères de l'auto-méditation}

1) L'auto-méditation entre dans le champ de ce que Husserl appelle la "réflexion égologique [Ichreflexion]» qui s'oppose radicalement à "l'expérience naturelle de soi-même [naturale Selbsterfahrung]»(App. XXIV, pp. 539-540). Cependant, elle occupe une place bien délimitée dans cette sphère de la réflexion égologique. Pour faire comprendre cette délimitation, le phénoménologue fait appel à une distinction entre «un concept plus large et un concept plus étroit de la méditation de soi-même [Selbstbesinnung] »(App. XXVIII, p. 566). La "réflexion égologique" correspond au concept large de l'auto-méditation en tant qu'elle est "réflexion sur l'ensemble de la vie de l'ego en tant qu'ego », alors que l'« auto-méditation " proprement dite correspond au concept étroit d'une "méditation au sens prégnant de la question-en-retour [Rückfrage] sur le sens de l'ego, sur son essence téléologique " (App. XXVIII, p. 566 ; cf. aussi App. XXIV, p. 539). L'auto-méditation phénoménologique s'apparente ainsi à la réflexion égologique, à la prise de conscience en général qui caractérise la méthode phénoménologique, avec cette précision, cependant, que le retour à la source du sens prend une dimension historique et critique tout en étant corrélatif à l'intérêt pour son accomplissement dans le devenir personnel. Dans la Krisis. . ., Husserl parle surtout de la méditation-de-soi au sens étroit, alors que, dans ses autres œuvres, il est plus souvent question de la "réflexion ego-logique » en tant qu'analyse des prestations de sens issues de la subjectivité transcendantale. Nous nous attacherons donc à ce concept étroit de l'auto-méditation qui devrait, selon Husserl, nous permettre de renouer avec le sens, corollairement de régler la crise de la perte du sens, en saisissant et en actualisant l'essence téléologique de notre vie. D'autre part, c'est l'adoption de l'épochè ou de «l'attitude personnelle» qui rend possible 
l'auto-méditation (App. XX, p. 523 ; App. XXIV, p. 539). Celle-ci apparaît même comme le recto de l'épochè, qui a souvent été décrite comme un moment négatif de l'expérience, comme un mouvement de rupture par rapport à l'attitude naturelle. Dans cette perspective, elle se présente comme un "apprendre-à-se-connaître transcendantal » (App. XX, p. 524), par opposition au se-connaitre-soi-même de façon naturelle. Elle est inconcevable sans cette perspective d'une attitude transcendantale transformant l'attitude naturelle ; Husserl soutient que le «transcendantal » désigne le motif originel de l'auto-méditation entreprise par l'Ego ( $\$ 26$, p. 113). Cette méditation se présente donc comme un questionnement réflexif sur le sens de notre vie, un peu à la manière de la méditation au sens où nous l'entendons couramment. Restent à préciser les dimensions « historique » et «critique » de l'auto-méditation.

2) En tant que retour historique ${ }^{10}$ sur le sens téléologique de la philosophie, elle remet en mémoire, dans l'esprit des philosophes qui méditent, les buts authentiques de la philosophie dont ils sont les héritiers, c'est-à-dire « ce qui, originellement et à chaque fois, a été voulu en tant que philosophie et a continué à être voulu à travers l'histoire dans la communion de tous les philosophes et de toutes les philosophies» $(\$ 7$, p. 23) ${ }^{11}$. Par elle, il est possible de retrouver et de se "présentifier »les intentions originaires du projet philosophique, qui se sont constituées dans la chaîne historique des philosophes à partir des fondateurs, ceux que Husserl appelle nos « ancêtres en philosophie [Urväter] " ou les "patriarches [Erzväter] (App. V, p. 433). Cependant, l'intérêt du philosophe méditant ne se dirige pas uniquement sur la volonté des premiers philosophes et sur celle de tous leurs successeurs passés ou actuels, il se dirige aussi sur sa propre volonté en tant qu'il est relié intentionnellement à la tradition philosophique; il est alors question pour ce dernier d'un « retour sur

10. Le caractère historique de la méditation est décrit au $\$ 15, \mathrm{p} .81-83$, à l'app. V, p. 433-434 ainsi qu'a l'app. XXVIII, p. 563-568.

11. L'ensemble de la Krisis... se présente comme une telle médication historique sur le sens téléologique de l'histoire philosophique. D'autres ceuvres husserliennes, également, témoignent d'un tel type de réflexion historique ou «archéologique », par exemple Philosophie Premiere 1, le début des Méditations Cartésiennes ainsi que certains passages des Reiberibes Logiques et de Logique formelle et logique transiendantale. 
ce vers quoi proprement s'élance sa volonté, sur ce qui est en lui volonté à partir du vouloir et en tant que volonté de ses ancêtres, c'est-à-dire de ses pères-en-esprit $\gg(\$ 15 \text {, p. } 83)^{12}$. Celui qui médite se rattache essentiellement à la connexion des philosophes qui forment une communauté particulière. Implicitement, son questionnement en retour se tourne vers ce que l'on pourrait appeler une intersubjectivité bistorique immanente, dans laquelle il « réactive » le sens originaire de la philosophie afin de pouvoir l'actualiser.

3) D'autre part, la méditation-en-retour (Rückbesinnung) n'est pas un pur et simple retour historique en tant que reprise naïve des buts de la philosophie, elle est également critique, c'est-à-dire qu'elle implique un "examen critique de ce qui, dans la détermination du but et dans la méthode, dénote cette ultime authenticité d'origine qui, une fois aperçue, contraint apodictiquement le vouloir $»(\$ 7$, p. 24$)$. Le questionnement critique réanime les buts authentiques de la philosophie, qui se sont sédimentés dans le cours de l'histoire. Il permet la répétition de leur fondation originelle dans un mouvement de reprise qui, en même temps, les clarifie et les corrige, afin de retrouver ou de conserver leur authenticité originaire ${ }^{13}$. Ainsi, la méditation cherche à s'assurer de l'authenticité des intentions originaires des patriarches de la philosophie, tout en essayant de les perfectionner.

Dans ce moment de la critique, une règle guide l'" autopenseur [Selbstdenker]": il doit avoir "la volonté de se libérer de tous les préjugés" qui proviennent d'une «sédimentation traditionnelle » ( $\$ 15$, p. 83$)$. Il ne doit pas se contenter de recevoir passivement les buts que lui transmet la tradition philosophique ; il doit plutôt confirmer leur validité, transformer pour ainsi dire ce qui se présente sous la forme de «pré-jugés» en de véritables «jugements». Cette idée du questionnement-en-retour critique, typiquement d'inspiration cartésienne, a des affinités avec l'idée d'épochè (App. X,

12. En d'autres termes, cette méditation est effectuée "pour traiter notre présent philosophique sous l'angle de l'histoire de la philosophie" (App. V, p. 433 ; cf. aussi app. XXVIII, p. 565-568).

13. Cette conception de la méditation critique et ce qu'elle peut accomplir est développée au $\$ 15$, p. $81-83$ et it l'app. V, p. $433-435$. 
p. 469), ce suspens critique à travers lequel la validité des choses est éprouvée. Cependant, dans la Krisis..., elle s'applique à l'histoire de la philosophie et, d'une manière nouvelle chez Husserl, elle s'applique également à notre histoire personnelle.

Car le questionnement critique s'exerce également sur le remplissement des buts de la philosophie dans le devenir personnel, individuel et communautaire. Dans le contexte d'un dépassement de l'auto-méditation des ancêtres, Husserl parle d'une "critique responsable [qui] a sa nature propre et trouve son terrain dans de telles déterminations bistoriques des buts personnels, dans la façon dont ses buts ont été relativement remplis, dans la réciprocité de la critique, et non dans les auto-évidences privées d'un philosophe actuel» ( $\$ 15$, p. 83). Nous remarquons, ici, une référence à ce qu'on pourrait qualifier de pratique intersubjective de l'auto-méditation, qui serait parallèle à l'idée d'une intersubjectivité bistorique immanente.

L'auto-méditation, en tant que questionnement historique et critique, cherche donc à saisir le sens, les buts authentiques, que les patriarches ont voulu donner au projet philosophique. Elle cherche à clarifier, à corriger et à perfectionner ces buts. Elle se tourne également vers leur remplissement pour en vérifier l'adéquation. Avant de s'engager plus profondément à décrire et à définir l'auto-méditation dans ses processus caractéristiques, tentons d'abord de cerner les buts qu'elle vise. En ayant ceux-ci à l'esprit, il nous sera plus facile, lorsque nous analyserons les opérations caractéristiques de l'auto-méditation, de vérifier si elles sont aptes véritablement à les atteindre. Il faut d'abord distinguer les buts immédiats des buts lointains, tout en explicitant comment la réalisation des buts immédiats entraine l'accomplissement adéquat des buts lointains.

\section{BUTS DE L'AUTO-MÉDITATION}

\section{A) Comprendre le sens de la tâche philosophique}

Le phénoménologue attend principalement de la méditation-en-retour de pouvoir se comprendre lui-même dans son 
existence philosophique (App. XXVIII, p. 566) ${ }^{14}$, et, par le fait même, de pouvoir saisir le sens et la tâche de la philosophie, à laquelle il devra contribuer, afin d'accomplir ses buts authentiques. Cependant, la compréhension du sens de la philosophie n'est pas la description objective de l'histoire de la philosophie ou celle de l'histoire personnelle du philosophe. Elle correspond plutôt à une compréhension de la direction téléologique que prend la philosophie dès ses débuts et qui s'offre aux philosophes comme une tâche à accomplir:

Il convient donc que nous nous donnions la compréhension de la téléologie dans le devenir historique de la philosophie, en particulier de celle des modernes, et du même coup que nous nous mettions au clair sur nous-mèmes en tant que nous sommes les porteurs de cette téléologie et que notre dessein personnel est de contribuer à l'accomplir $(\S 15, \text { p. } 81)^{15}$.

Par cette compréhension, celui qui médite est en mesure de " maîtriser le sens » véritable de la philosophie afin de mieux le réaliser : "me rendant maitre du commencement originel du sens qui travaille l'histoire, et m'enfonçant dans ce sens (dans l'intentio), je puis accomplir une interprétation orientée sur des possibilités eidétiques de remplissement de cette intention » (App. V, p. 434).

Le phénoménologue peut envisager ce processus d'une maîtrise du sens en vertu de sa vision schématique de l'intentionnalité structurant l'expérience historique: la visée intentionnelle appelle un remplissement; la philosophie contient une telle visée intentionnelle dont il s'agit de comprendre le sens afin de le remplir. Cependant, pour Husserl, cette compréhension ne consiste pas simplement à actualiser une philosophie du passé, il faut que l'idée téléologique de la philosophie se renouvelle grâce au travail des philosophes contemporains qui doivent, à cet effet, modifier, ajuster er perfectionner l'intention philosophique ( $\$ 56$, p. 216 ; App. V, p. 434-435). D'autre part, ce but de

14. "Il est clair (d'où, sinon, pourrait nous venir le secours ?) que nous avons besoin de la pénétration d'une méditation-en-retour, histarique et critique, afin de nous soucier d'une compréhension radicale de nous-mêmes avant toute décision " $(\$ 7$, p. 23)

15. Le $\$ 56$ développe largement cette idée par rapport à celle d'un avènement historique de la philosophie transcendantale en tant que "science fondatrice en dernière instance et universelle $"$. 
l'auto-méditation déborde largement les préoccupations individuelles du philosophe. Car la tâche prescrite par la philosophie concerne l'humanité entière. Le philosophe est responsable non seulement de lui-même, mais aussi de tous les autres hommes : "C'est seulement dans cette plus haute conscience de soi, qui devient elle-même l'un des rameaux de la tâche infinie, que la philosophie peut remplir sa fonction, de se mettre elle-même sur la voie et, par là, d'y mettre une humanité authentique " (Annexe III, p. 373). Nous aurons à nous interroger sur cette lourde responsabilité qu'Husserl assigne aux philosophes, «fonctionnaires de l'humanité » $(\S 7, \text { p. } 23)^{16}$.

Mais quelle est précisément la tâche qui incombe à la philosophie? Quelle est sa fonction parmi toutes les entreprises humaines? Disons d'abord que, selon Husserl, il revient à la phénoménologie, seule, d'avoir mis à jour le sens ultime de cette tâche, en tant que la philosophie doit devenir une auto-méditation qui se rend responsable, de façon ultime, d'un devenir authentique ou qui assume la fonction de "rendre possible un Dasein humain rempli de sens » (App. X, p. 472). Cette tâche est exprimée de diverses manières ${ }^{17}$ : le plus souvent, le phénoménologue parle de constituer une humanité selon la raison; il est alors question de "donner une nouvelle figure, à partir de la libre raison, à partir des vues théoriques d'une philosophie universelle, non seulement à soi-même éthiquement, mais à l'ensemble du monde humain ambiant à l'existence politique et sociale de l'humanité $»(\$ 3, \text { p. } 12)^{18}$.

16. "Le philosophe comme "fonctionnaire de l'Humanité "signifie qu'il est professionnellement destiné à définir et à rendre conscientes les conditions d'une humanité, c'est-à-dire de la participation de tous à une vérité commune. "

Maurice Merleau-Ponty, Les sciences de lhomme et la phénoménologie, Paris, Centre de documentation universitaire, 1966, p. 2

17. Nous ne nous attarderons pas sur la tàche générale que Husserl assigne à la philosophie, qui est de garantir la vérité de nos ronnaissances et de guider l'homme dans son entreprise d'expliciter l'Univers: "D’après cette idée, la philosophie devrait être une connaissance fondée sur un suprême et ultime effort de compréhension (Selbstverständigung) et de justification de soi-même (Selbstverantwortung), qui devait à chaque instant guider le sujet connaissant dans ses actes de connaissance."

Edmund Husserl, Philosophie Première, Deuxième partie, trad. par A.L. Kelbel, Paris, P.U.F., 1972, p. 3-4. À l'avenir, nous référons au titre de cet ouvrage par l'abréviation : P.P.2.

18. Plusieurs textes expriment de manière analogue cette tâche de la philosophie : $\$ 6$, p. $21 ; \$ 56$, p. 223. 
Parfois, la philosophie est envisagée comme le moyen de rendre autonome et d'émanciper l'bumanité, en visant "l'auto-création [SelbstSchöpfung] de son être véritable (App. X, p. 472 ; cf. aussi $\$ 73$, p. 302). On doit comprendre aussi que la philosophie s'est déjà engagée dans cette tâche très lourde de créer une civilisation de la raison; à cet effet, l'humanité européenne lui doit son visage. Husserl pense que l'auto-méditation met le philosophe en position de continuer à assumer cette tâche et de la mener à terme.

\section{Remarques sur le cadre téléologique de l'auto-méditation}

Deux remarques nous viennent à l'esprit relativement à ce but de l'auto-méditation, qui vise à saisir le sens de la tâche philosophique. 1) Husserl nous propose implicitement, avec l'idée d'auto-méditation, que c'est par un "se connaitre soi-mêtme » que nous pouvons espérer un devenir personnel selon la raison. Ainsi, la "Selbstbesinnung " prolonge la tradition philosophique du "Connais-toi, toi-même" par lequel Socrate espérait une sagesse universelle. Car elle se présente comme une «connaissance de soi » qui implique, en elle-même, une réalisation authentique de toute l'humanité :

Tout ceci implique que ce niveau le plus élevé de l'humanité, celui d'une existence de l'humanité (et non d'un quelconque Dasein humain individuel abstrait), soit une existence dans la volonté apodictique de suivre des buts entièrement apodictiques, existence dont la fonction est justement l'auto-méditation, la connaissance universelle apodictique de soi-même, et corrélativement du monde, en tant que forme nécessaire. en tant que condition de possibilité pour la réalisation d'une existence authentique de l'bumanité [nous soulignons] dans un devenir authentiquement "humanitaire», dans une authentique destination, voulue ainsi apodictiquement et qui a triomphé de l'aveugle Moira (App. X, p. 475).

L'auto-méditation traduit ainsi une volonté de fonder toute entreprise humaine sur la connaissance et de répondre rationnellement de son existence.

2) Cette première remarque nous permet de mieux saisir le schéma téléologique dans lequel la méditation s'insère. Cette dernière s'articule dans une téléologie de la raison qui allie son 
propre mouvement de clarification à celui de son effectuation. Au fur et à mesure que le sujet éclaircit en lui, par sa méditation, la raison qui donne leurs sens à toutes ses expériences, il parvient progressivement à la possibilité de remplir les tâches de la raison. La méditation, qui part de l'immanence du sens dans la subjectivité méditante, accède à sa transcendance et à son accomplissement effectif par la médiation de l'intersubjectivité. Celle-ci est en quelque sorte la charnière essentielle de tout le schéma, car c'est en essayant de rendre intersubjectif même omnisubjectif - l'éclaircissement du sens rationnel en lui que le sujet méditant peut envisager son accomplissement dans son propre devenir et dans celui de tous les hommes $(\S 73$, p. 301-306) ${ }^{19}$. Les quelques traits de ce schéma confirment l'idée d'une réalisation de l'être raisonnable de l'homme impliquant l'entrée en jeu et le développement de l'auto-méditation. Ils nous apprennent également que l'auto-méditation est l'occasion pour la Raison immanente d'effectuer un « retournement » sur elle-même, de telle sorte qu'elle se rend responsable de son propre accomplissement au niveau transcendantal : "la raison cachée et non déployée devient la raison qui se comprend et se règle elle-même. Il fait essentiellement partie du sens de toute raison qu'elle soit, en un sens inévitablement large, raison connaissante. Elle est partout méditation responsable sur ce qui est vrai ou faux $\gg$ (App. X, p. 475$)^{20}$. Le schéma implique également que la méditation,

19. "Se vouloir comme raison, c'est vouloir l'avènement universel de la raison en tous et chacun, cest donc vouloir non seulement un savoir universel apodictique, mais aussi une société universelle de la réciprocité absolue. "

Jean Ladrière, Vie sociale et destinée, Belgique, Éd. J. Duculot, 1973, p. 33.

20. Plusieurs interprètes de Husserl ont retenu cette idée :

- "Prendre conscience (besinnen) des origines, c'est en même temps se rendre responsable (verantworten) du sens (Sinn) de la science et de la philosophie ; c'est l'amener à la clarté de son "remplissement", c'est se mettre en mesure d'en répondre à partir du sens de la totalité de notre existence. "

Jacques Derrida, "Introduction " à : Husserl, l'origine de la gémétrie, Paris, P.U.F., 1974, p. 11.

- «a raison n'est pas seulement l'effort, en nous, pour comprendre la totalité et en déchiffrer le sens, c'est aussi le pouvoir qui nous rend responsables de nous-mêmes et l'effort par lequel nous tentons de nous égaler à l'exigence que cette responsabilicé rend manifeste."

Jean Ladrière, Vie sociale et destinée, p. 10.

- "Le devenir est formé des degrés que parcourt la raison pour se réaliser et se rendre responsable d'elle-même."

Walter Biemel, «Introduction" à : Husserl, "la philosophie comme prise de conscience de l'humanité ", trad. par P. Ricour, dans Dewalion 3. Vérité et liberté. Neuchâtel, Ed. de la Bacconière, 1950, p. 114. 
en rendant manifeste à tous que la Raison oriente le destin de l'humanité, fait en sorte que cette destinée soit assumée de plus en plus explicitement par tous les hommes.

\section{B) Réaliser l'être authentique de l'homme}

On doit tenir compte de ce que le questionnement réflexif intervient à l'intérieur d'une téléologie rationnelle pour comprendre adéquatement de quoi elle est responsable, c'est-à-dire ce que sont ses buts ultimes. Ces buts sont: 1) que l'Ego réalise son être véritable et 2) que l'humanité atteigne son être authentique:21 «L'auto-méditation reste la fonction constante du devenir pleinement homme et de la réalisation d'une pleine humanité » (App. XXIV, p. 539). La méditation cherche aussi, dans une perspective pratico-éthique, à connaître la façon dont l'humanité peut devenir "heureuse " (App. XIX, p. $518 ; \S 73$, p. 304), ce qui sous-entend que l'homme réussirait alors à vivre selon la raison. Le Selbstbesinnung se revèle progressivement comme un vieil idéal caractérisant assez bien l'histoire de la philosophie, du moins en deçà d'une certaine "clôture " dans les limites de laquelle la Raison peut imposer son joug à l'existence humaine, peut «ar-raisonner » la vérité et l'homme.

En tenant compte du cadre téléologique, dans lequel apparaît l'auto-méditation, et des buts ultimes qui appartiennent à la tâche originelle de la philosophie, il nous reste à nous interroger sur son efficacité réelle pour atteindre ces buts. Peut-elle vraiment permettre à l'homme de se comprendre et de se réaliser d'une façon authentique? En réactivant les buts originaires des ancêtres-philosophes qui ont auvré à la fondation de l'humanité occidentale, peut-on espérer que ceux-ci puissent motiver non seulement la constitution d'une nouvelle figure de l'humanité, mais encore la réalisation d'une "humanité authentique ", avec un sens valable universellement? Quelle est donc la véritable efficacité de l'auto-méditation?

21. La formulation de ces buts apparaît au $\$ 73$, p. $301-302$ et aux app. XXIV, p. 538 , app. X, p. 475. 


\section{LE PROCESSUS DE L'AUTO-MÉDITATION}

La réponse à ces questions exige d'abord qu'on se représente schématiquement le processus de l'auto-méditation ; celui-ci se divise en trois opérations intimement liées entre elles: 1) la reprise critique des buts originels des ancêtres-philosophes ; 2) la correction critique de ces buts au cours de leur réalisation dans le devenir personnel ; 3) l'inter-subjectivisation de l'auto-méditation, c'est-à-dire sa transmission à l'ensemble des hommes et son exercice dans une intersubjectivité transcendantale.

1) Le questionnement historique de la méditation implique une reprise critique du projet philosophique. Comme opération, cette reprise s'apparente à une évocation, à une "remise en mémoire " des buts de la philosophie. Husserl parle alors de l'auto-méditation qui "éveille la mémoire historique » de nos ancêtres-philosophes, ou encore qui opère une "pré-sentification" des intentions originelles de la philosophie dans le souvenir historique (App. V, p. $433 ; \S 15$, p. 83). La méditation historique est présentée comme une réativation du sens originaire de la philosophie, un peu à la manière de la réactivation des premiers acquis de la géométrie, décrite dans l'Origine de la géométrie. Cependant, ce réveil des intentions originelles de la philosophie n'est pas une opération naïve, mais un retour aux buts des ancêtres-philosophes avec une attitude questionnante. Il implique une opération critique qui met à jour et vérifie, à travers les couches sédimentées des modifications de l'intentionnalité, celle qui est originaire (App. V, pp. 434-435). Il est important de noter, cependant, que cette évocation ou réactivation impliquant une clarification critique se déroule d'abord exclusivement au niveau intrasubjectif. En général, les premiers mouvements de la méditation sont d'abord aperçus dans une perspective purement intrasubjective. Leur dimension intersubjective se manifeste par la suite.

2) Le philosophe qui médite, en plus de retourner aux buts premiers de la philosophie, s'emploie également à les corriger. Cette opération est imbriquée très étroitement dans la première. Elle prolonge et dépasse la simple reprise en cherchant à réaliser les buts qui se rattachent à la tâche philosophique. La 
méditation permet alors de corriger un « remplissement imparfait " en visant toujours un remplissement de plus en plus parfait (App. V, p. $434 ; \S 15$, p. 83). Cette « correction" correspond à un deuxième sens de la critique qui se préoccupe davantage de vérifier l'adéquation de la tâche prescrite avec son remplissement. Et comme nous l'avons remarqué précédemment, il y a correction non seulement des buts saisis, mais aussi de la direction actuelle de leur remplissement, afin d'accomplir l'intention originaire de la philosophie dans l'existence humaine. Ainsi la « méditation critique » vérifie, guide et réajuste le mouvement de cet accomplissement. Par sa première opération, la méditation se tourne vers la signification historique de la tâche philosophique, vérifiant son authenticité d'origine : par sa deuxième, elle se tourne vers son accomplissement dans l'existence historique des hommes, tout en effectuant les corrections appropriées pour obtenir un remplissement adéquat. La critique opérée par le questionnement réflexif constitue l'instance de transition entre les buts énoncés dans la théorie philosophique et leur accomplissement dans la pratique existentielle, celle-ci pouvant être soumise d'une manière constante au jugement critique $^{22}$. Le sens de notre existence est assimilé par Husserl à un "héritage " que nous assumons et à une "tâche " qui nous est confiée. Le phénoménologue nous convie à développer en nous une "compréhension critique de l'unité d'ensemble de l'histoire, de notre histoire» ( $\$ 15$, p. 82$)$, une comprébension critique de l'béritage d'une humanité issue de la raison philosophique et de la tâche qui pourrait rendre cette humanité à la Raison. Ces concepts d'héritage et de tâche impliquent également que la saisie clarifiante et le remplissement critique de l'intention originelle s'effectuent dans les contextes intersubjectifs et omnisubjectifs. Nous héritons d'une tâche. Cet héritage renvoie à une générativité intersubjective, tandis que la tâche à remplir renvoie à une coexistence intersubjective des

22. L'annexe III envisage d'une façon similaire la "critique ", qui correspond alors à une "synthèse des deux sortes d'intérêts qui s'accomplit dans le passage de l'attitude théorique à l'atrirude prarique, de manière que la théoria qui forme une unité close et qui se déploie dans l'épochè de toute pratique (la science universelle donc) ait pour vocation [. . . ] de servir d'une nouvelle façon l'humanité, et tout d'abord celle qui vit dans l'expérience concrète, et d'une façon qui continue toujours à être naturelie» (Annexe III, p. 363). 
philosophes. Ce contexte nous introduit à une troisième opération de l'auto-méditation, soit l'opération intersubjective.

3) Il faut préciser que l'intersubjectivité ne fait pas que s'additionner aux opérations de la reprise et de la correction, mais qu'elle les caractérise essentiellement, tout en étant une nouvelle opération de l'auto-méditation extrêmement importante afin que celle-ci accomplisse efficacement ses fonctions. Il faut donc expliciter comment l'auto-méditation implique une opération intersubjective qui est inhérente à la reprise et à la correction des buts originels de la philosophie par le sujet méditant, et qui semble essentielle à la réalisation adéquate de ces buts dans l'existence du philosophe et de l'humanité.

A) D'abord, la reprise elle-même de l'intention originelle des premiers philosophes correspond à une opération intersubjective. En fait, il s'agit ici d'un retour à une intersubjectivité historique ; cela signifie que le questionnement-en-retour, relevant d'un effort purement subjectif, s'oriente vers une activité intersubjective, instituée dans l'histoire entre les philosophes, et à travers laquelle s'est constitué le projet «philosophie ${ }^{23} »$. Ce questionnement consiste alors à « réveiller la chaîne des penseurs, leur socialité de pensée, leur communauté idéelle [Gedankliche Vergemeinschaftung], afin de la transformer pour nous en un présent vivant $»(\S 15, \text { p. } 83)^{24}$. Ainsi celui qui veut reprendre les buts originels entre en connexion avec une chaîne intersubjective formée par l'échange des savants entre eux ; d'une certaine façon, il s'assimile à cette chaîne tout en l'intégrant à sa propre conscience réflexive. La connexion intersubjective de la communauté historique des philosophes, qui se questionnent et se répondent mutuellement, se réveille alors dans sa conscience. Cependant, cette opération ne se réduit point à un ressouvenir immanent élaboré dans une conscience solipsiste. Tandis que le ressouvenir «présentifie» les situations et les intentions propres du moi, l'auto-médita-

23. "L'existence-comme-philosophe, en tant qu'existence par la générativité philosophique, comprend dans son sens même la question historique-en-retour sur cette générativité même, autrement dit sur sa tradition propre "(App. XXIV, p. 543 ; cf. aussi app. XIIl, pp. 489-492).

24. Cette idée d'une médiration-en-retour en tant que saisie de l'intersubjectivité historique dans laquelle se constituent les buts de la philosophie se retrouve aux app. XXIV, pp. 540-543 ; app. XXV, p. 548 ; app. XXVIII, p. 566-568. 
tion »présentifie » d'abord les intentions des autres telles qu'elles se présentent dans le nexus intersubjectif, de telle sorte que le philosophe méditant, connecté à ce même nexus, est en mesure de les faire siennes, de les modifier, etc.. Plusieurs expressions marquent les aspects de cette communauté intersubjective vers laquelle aboutit le questionnement réflexif: "société des philosophes" (App. V, p. 548), « communion de tous les philosophes " ( $\$ 7$, p. 23$)$, "générativité " et " coexistence philosophique " (App. XXIV, p. 542), ou encore «connexion philosophico-historique» (App. XXV, p. 544; App. XXVIII, p. 568). Cette idée d'un réveil de l'intersubjectivité est également motivée par le fait que la connexion peut parfois se rompre, et que la générativité philosophique ne se propage pas toujours avec la même force (App. XXV, p. 542). La méditation vient comme rétablir cette générativité intersubjective. Et ce rétablissement est rendu possible par la méditation des documents philosophiques; les idées, les intentions, qui y sont consignées peuvent être réactualisées : "Mais les idées devenues ainsi des documents, des sédiments, peuvent $/ \ldots$ / se remettre à vivre " (App. XXIV, p. 542). La méditation, d'une certaine façon, répare l'intersubjectivité rompue momentanément. D'une façon générale, l'automéditation opère sur les "documents littéraires", sur les discours philosophiques sédimentés dans des textes (App.XXIV, p. 542-543; App. XIII, p. 490-491; App. XXVIII, p. 566-568)25. Celui qui médite ces textes est en mesure de réactiver les intentions originelles constituées dans l'échange philosophique, de comprendre "le projet commun à tous » et de le reprendre. Cette réactivation et le rattachement à la communauté intersubjective qu'elle implique signifient que les «formations idéales de la Théoria sont, tout simplement, vécues à plusieurs et reprises en commun pour la répétition de leur compréhension et de leur production" (Annexe III, p. 367). Dans l'Origine de la géométrie, Husserl

25. "À partir du document reçu et déjà lisible, la possibilité m'est offerte d'interroger à nouveau et en retonr sur l'intention originaire et finale de ce gui m'a été livré par la tradition."

Jacques Derrida, "Introduction " à : Husserl, rorigine de la géométrie, p. 36.

En général, cette "Introduction " est une interrogation essentielle sur les rapports entre la tradition historique et l'écriture. 
exemplifie largement cette idée d'une reprise des acquis antérieurs comme rattachement à une intersubjectivité par l'intermédiaire des textes qu'il faut réactiver. En méditant sur les énoncés de la géométrie, il est possible de réanimer les intentions originelles des proto-gémètres, de reconstruire les premiers acquis, qui s'offrent à nous sous la forme d'objectivités dont nous héritons, et à partir desquelles nous en produisons d'autres. L'héritage et la tâche de la géométrisation du monde se gagnent par une méditation sur les documents de la géométrie; de la même manière, on gagne l'héritage et la tâche d'une rationalisation philosophique de l'existence humaine en méditant sur les documents où s'inscrit le message du Logos philosophique. La Raison s'exprime et s'imprime dans le discours philosophique; l'auto-méditation réveille l'esprit de l'écriture. Les textes philosophiques forment donc le lieu idéal où la faculté de réactivation et la «critique documentée » (App. XXV, p. 544) peuvent s'exercer, éclaircissant et corrigeant les énoncés produits par l'histoire de la philosophie, qui portent sur la vérité du monde ainsi que sur l'authenticité de notre existence. De cette façon, la méditation sur le discours philosophique sédimenté dans des documents littéraires correspond au réveil, en soi-même, d'une intersubjectivité historique.

B) Par ailleurs, la correction implique aussi une opération intersubjective: à partir de la présentification de la communauté idéelle, l'auto-méditation exerce une «critique responsable», dans la "réciprocité » entre les auto-penseurs ( $\$ 15$, p. 83 ; Annexe III, p. 367-368). Cependant, il ne s'agit plus ici de réveiller une intersubjectivité historique dans la seule conscience du philosophe méditant, mais d'entrer pratiquement dans cette connexion intersubjective, pour que les intentions soient comprises par une communauté de philosophes qui se corrigent mutuellement. Il est alors question d'une méditation responsable qui assume, dans une vérification communautaire, la visée des buts originels et leur remplissement. Ce caractère "responsable » de la méditation critique ${ }^{26}$ indique

26. Il faudrait noter différents sens que Husserl donne à cet important concept de "responsabilité " dans son aeuvre. Déjà dans la Krisis . . . trois sens apparaissent :

1) la responsabilité épistémologique, selon le modèle carrésien, par laquelle le philosophe 
précisément qu'elle doit devenir une praxis intersubjective, renouvelant la société des philosophes: «Manifestement sa méditation [celle du philosophe contemporain] doit prendre le caractère de la responsabilité radicale, et certes d'une responsabilité qui se joue dans sa propre personne, mais qui revêt cependant le caractère d'une responsabilité communautaire, celle de l'ensemble de la communauté philosophique contemporaine» (App. XXIV, p. 543). L'auto-méditation rend d'abord le philosophe responsable d'entrer en connexion intersubjective avec les autres, puis, à travers celle-ci, de poursuivre, rectifier, perfectionner les buts du projet philosophique. De cette façon, on doit comprendre que la correction légitimante des buts est corrélative à une méditation devenant la responsabilité d'une communauté. Ainsi, l'auto-méditation implique, dans son processus même, son élargissement vers une méditation intersubjective: "Il est bien entendu que donc en tant que philosophe je ne veux et ne peux vouloir rien d'autre que des prises de conscience [Selbstbesinnungen] radicales de moi-même qui du fond d'elles-mêmes se transforment en prises de consciences de soi de l'intersubjectivité existant pour moi ${ }^{27}$.

L'intersubjectivité apparaît comme le corrélat immédiat de l'auto-méditation : d'abord, lorsqu'elle est éveillée dans la conscience immanente de l'archéologue, puis lors de la correction critique des buts de la philosophie et de leur accomplissement dans le devenir même de l'humanité ; il est alors question de parvenir à une intersubjectivité transcendantale en élargissant la communauté effective de ceux qui méditent, ou encore de propager jusqu'aux confins de l'humanité non seulement les buts de la philosophie, mais également

doit répondre de la validité des connaissances en s'appuyant sur un fondement apodictique.

2) la responsabilité éthique, comme l'entend Kant, où le philosophe cherche à répondre de son comportement d'une manière universelle.

3) La responsabilité intersubjettive, impliquée par l'idée de l'auto-méditation, qui apparaît à première vue comme une tentative d'envisager la fondation objective des connaissances et la fondation universelle du devenir personnel au niveau d'une communauté intersubjective qui transcende la fondation subjective.

27. Edmund Husserl, Logique formelle et Logique transiendantale, trad. par S. Bachelard, Paris, P.U.F., 1965, p. 366. À l'avenir, nous réféterons au titre de cet ouvrage par Pabréviation L.F.T. 
l'attitude avec laquelle on peut soi-même les comprendre et les réaliser en liaison avec les autres, de telle sorte qu'on parviendrait au règne d'une société universelle de philosophes produisant des vérités et des normes objectives, qui assureraient l'harmonisation de l'humanité.

C'est en retraçant "l'origine historique de l'humanité philosophique et scientifique" (Annexe III, p. 356) que le phénoménologue aperçoit le style essentiel du développement et de l'élargissement d'une communauté harmonieuse des hommes répondant apodictiquement de leur devenir personnel, individuel et communautaire, lequel style, d'autre part, peut être transmis plus ou moins explicitement à l'ensemble des hommes qui n'appartiennent pas encore à la république des philosophes. Le processus dessiné par ce modèle se déroule comme suit : avec la première conception des idées, le premier philosophe s'éveille à un « nouveau style de l'existence personnelle » qui se transmet aux autres. C'est ainsi que se constitue la communauté des "philosophes"dont les membres collaborent à la «production des idées et à la normation idéale de la vie » (Annexe III, p. 356). Cependant, cette dernière ne se constitue véritablement que dans la mesure où certains hommes adoptent "l'attitude théorique " et mettent en suspens «l'attitude naturelle» (Annexe III, p. 360). Progressivement, l'esprit de cette nouvelle communauté, dont on retrace les origines dans la Grèce antique, s'étend à l'ensemble de l'humanité européenne. L'élargissement de la philosophie même se produit de deux manières : "En tant qu'extension d'une communauté de philosophes par vocation et en tant que mouvement de culture coextensif à la première " (Annexe III, p. 367). D'une part, les non-philosophes qui s'attachent à comprendre l'activité communautaire des philosophes deviennent eux-mêmes des philosophes ; d'autre part, la philosophie exerce sa "fonction archontique" vis-à-vis de l'ensemble de l'humanité, elle gouverne son destin. À cet effet, elle produit une nouvelle praxis, la praxis critique, qui «vise à élever l'humanité grâce à la raison scientifique universelle suivant des normes de vérité de tout type, qui visent à en faire une humanité fondamentalement nouvelle, capable de répondre absolument d'elle-même sur le fondement de vues théoré- 
tiques absolues»(Annexe III, p. 363). En interrogeant ainsi les débuts de la philosophie, l'archéologue aperçoit la constitution d'une communauté intéressée à produire, dans un travail commun et dans une attitude théorique, une science universelle qui pourrait répondre de toute connaissance et du devenir même de l'humanité. Il aperçoit aussi que cette communauté trouve la possibilité de réaliser son idéal en le propageant aux autres et en s'élargissant comme communauté à la grandeur de l'humanité.

Par ailleurs, l'auto-méditation implique d'autant plus une pratique intersubjective s'élargissant universellement, que la réalisation des buts de la philosophie dans le devenir personnel ne peut être envisagée sans la corrélation nécessaire entre l'individu et tous les autres: "si l'homme est un être raisonnable (animal rationale) il ne l'est que dans la mesure où toute son humanité est une humanité raisonnable » $(\$ 6$, p. 21). Cet être raisonnable de l'homme s'obtient à un certain degré du développement de la vie personnelle à travers les étapes d'une auto-méditation responsable s'exerçant d'abord occasionnellement, puis universellement, par laquelle l'Ego prend la résolution de se réaliser selon la Raison,

mais cela en corrélation indissoluble avec les personnes individuelles et les communautés, grâce à leur liaison interne immédiate et médiate dans toutes les formes de l'intérêt - qui sont liées dans l'harmonie ou dans la lutte - et dans la nécessité de ne conduire la raison personnelle individuelle à une réalisation toujours plus parfaite que comme raison personnelle communautaire, et inversement $(\$ 73$, p. $301-$ 302).

Arrêtons-nous un instant sur les résultats de notre analyse des opérations de la méditation phénoménologique. Deux idées retiennent notre attention :

1) Ces opérations impliquent d'abord l'effort individuel d'une subjectivité qui reconstitue en elle l'intersubjectivité originelle qui a constitué le projet philosophique, avec la volonté de remplir ses aspirations.

2) Parallèlement à cet effort subjectif de comprendre le sens de la tâche philosophique, s'amorce le mouvement constitutif d'une 
intersubjectivité transcendantale au sein de laquelle pourraient se réaliser les buts de la philosophie dans le devenir personnel, individuel et communautaire, étant entendu que la formation de cette communauté intersubjective coincide avec l'extension et le perfectionnement de l'auto-méditation en chacun de ses membres. En d'autres mots, notre analyse retient que l'automéditation, pour atteindre tous ses objectifs, amorce son processus par un retour " ego-logique " ve.s une intersubjectivité historique qui est intégrée dans le flux immanent de la conscience par la médiation du discours philosophique; elle découvre alors que c'est au sein de cette intersubjectivité que s'est constitué le projet de mettre à jour et d'accomplir la téléologie du logos rationnel en visant l'établissement d'une communauté transcendantale concrète, dans laquelle chaque membre exercerait l'auto-méditation, permettant ainsi une poursuite et un achèvement - sans obstacle, sans distorsion -, des buts ultimes de la philosophie.

\section{PROBLÈMES LIÉS À L'IDÉE D'UNE AUTO-MÉDITATION INTERSUBJECTIVE}

Le renvoi de l'auto-méditation à l'intersubjectivité pose un problème de taille : comment la subjectivité méditante peut-elle répondre ou se porter garante de l'auto-méditation des autres, pour rétablir une intersubjectivité historique qui soit exempte de distorsion ou pour parvenir à une pratique intersubjective authentique et, ainsi, fonder une véritable communauté transcendantale? Une reprise et un accomplissement authentiques du sens originel de la philosophie étant liés à l'exercice de l'auto-méditation en chaque membre de la nouvelle communauté des philosophes, comment l'auto-méditation égologique peut-elle s'assurer de sa propre communisation?

\section{A) Réduction et reprise d'une méthode écrite}

En parlant d'une "extension ", d'un " transfert " à tous les hommes de l'auto-méditation en tant que méthode fondant ultimement toute compréhension et toute réalisation de soi-même, ne mésinterprétons-nous pas le sens authentique d'une communisation de l'auto-méditation? Peut-être n'avonsnous pas tenu compte suffisamment du caractère égologique de 
la méditation, corollaire de la méthode réductrice? Bien que nous ayons déjà caractérisé l'auto-méditation comme étant le "recto " de l'épochè, ne nous sommes-nous pas trop éloignés du cadre de la réduction? Nous examinerons donc plus attentivement ce cadre en essayant de voir comment l'automéditation peut s'assurer de sa propre communisation.

Effectivement, l'auto-méditation s'inscrit dans l'épochè et la réduction transcendantales : «l'épochè (. . .) rend possible une "méditation " universelle sur mon être égologique ", (App. XX, p. 523) ${ }^{28}$. Dans ce cadre, la méditation égologique, bien qu'elle soit opérée en moi, ne se confine pas à ma subjectivité, mais me fait accéder à l'intersubjectivité transcendantale $^{29}$, précisément sous la forme d'une «méditation universelle sur mon être égologique ». Cette communisation universelle de la méditation s'effectue de la manière suivante:

La pure auto-méditation de l'ego - que j'accomplis en tant qu'ego - conduit à moi en tant que personne parmi les personnes, en tant qu'ego de mon monde ambiant égologique, qui fait partie de moi en tant que ce dont j'ai conscience dans ma conscience, en tant que ce qui apparaît à moi et vaut pour moi, et elle conduit également, par la sym-pathie [Einfühlungl, aux co-personnes, en tant qu'elles sont conscientes d'elles-mêmes comme ego, peuvent se méditer elles-mêmes (App. XXIV, p. 540) ( $^{30}$.

De cette manière, l'auto-méditation opérée dans l'épochè me fait accéder à une connexion subjective et intersubjective où me sont données les synthèses qui intègrent et harmonisent les modifications intentionnelles. J'accède a priori à la totalité du monde en tant que phénomène appartenant à mon système constitutif. L'humanité étant impliquée dans ce phénomène du monde, je peux immédiatement rattacher tous les autres ego à ce même système constitutif ou à cette même structure de concordance par quoi le monde acquiert son sens et vient à

28. Dans L.F.T., il est question d' une prise de conscience de soi [Selbstbesinnung] que je commence avec la réduction transcentale » $($ L.F.T. \$104, p. 365; cf. aussi à P.P.2, leçon 52, p. 229).

29. Cf. à L.F.T., \$104, p. 366, déjà cité au $\mathrm{n}^{\circ} 27$

30. Husserl présente aussi cette communisation comme une connaissance de soi ultime qui distingue les autres ego pour ensuite établir une communauté avec eux de telle sorte que " la connaissance de soi-même devient bientôt "connaissance-(de)-nous " absolue, connaissance de lomnisubjectivité absolue, dans la concrétion de laquelle le monde se constitue comme monde-pour-tous, prise commune" (App. XX, p. 524). 
valoir objectivement. Tout cela implique que la communisation de l'auto-méditation ne correspond pas à une espèce de "transfert " empirique de la méthode d'un homme à l'autre ${ }^{31}$, car, alors, nous retomberions dans l'attitude nä̈ve.

Cependant, Husserl avoue qu'il y a une certaine "naiveté " à poser ainsi une communisation immédiate de l'automéditation, ou encore à " attribue[r] d'un seul coup et sans fondement à l'humanité dans laquelle je me trouve la même métamorphose qui en fait une subjectivité transcendantale fonctionnante, alors que je n'avais accompli cette métamorphose qu'en moi seul " ( $\$ 54$, p. 212). Pour fonder cette attribution immédiate, nous devons suivre scrupuleusement les exigences méthodologiques d'une épochè radicale: d'abord, dans ma sphère transcendantale, l'ego peut se décliner par une série de modifications intentionnelles, à commencer par mon " je " primordial avec sa sphère d'appartenance à partir de laquelle il accède à "autrui " (au «tu ») avec sa perception donnée en tant que "modification intentionnelle de soimême " ( $\$ 54$, p. 210$)$. Cet accès à l'expérience d'autrui se comprend en analogie avec la remémoration, car l'apprésentation du moi d'autrui dans l'intropathie s'effectue à la manière de la présentification de mon moi passé dans le ressouvenir, avec cette réserve que le moi d'autrui ne se confirme pas en moi de la même façon que mon moi passé. Ensuite, autrui étant impliqué dans mon ego, on parvient à la constitution d'une intersubjectivité transcendantale « dans laquelle j'entre à mon tour, mais alors en tant que "un » ego transcendantal parmi d'autres, et. de même " nous tous" en tant que fonctionnant transcendantalement $\gg(\$ 54$, p. 210$)$. Moi et les autres devenons alors les co-sujets d'une humanité qui « s'acquitte intentionnellement, dans la communisation, de la validation du monde " ( $\$ 52$, p. 199). Par cette double fondation de l'ego d'autrui et de tous les ego de l'intersubjectivité transcendantale dans mon ego, une réduction universelle devient possible. Elle me connecte à un enchaînement synthétique universel qui amène à la concordance autant le divers de

31. "L'épochè non seulement serait manquée par une réduction individuelle à l'intérieur des âmes individuelles, mais $[$. . ! elle serait manquée aussi si on la poursuivait d'àme en âme en tant que réduction individuelle $»(\$ 71, \mathrm{p} .288)$. 
mes expériences dans le champ de la temporalité que le divers de toutes les expériences dans le champ de l'intersubjectivité ${ }^{32}$.

Le phénoménologue résout donc le problème d'une communisation de l'auto-méditation en recourant à une radicalisation de l'épochè qui devient une méthode de fondation ultime, valable universellement, d'abord pour mon ego, mais aussi pour tout ego inclus déjà dans mon champ transcendantal et qui accède à son tour au même champ:

Du point de vue méthodologique, c'est seulement à partir de l'ego et de la systématique de ses fonctions et prestations transcendantales que peuvent être exibées l'intersubjectivité transcendantale et sa communisation transcendantale, dans laquelle "le monde pour tous" et pour chaque sujet $e n$ tant que monde pour tous se constitue à partir du système de fonctionnement des pôles égologiques ( $\$ 54$, p. 211).

C'est également au moyen de ce point de vue méthodologique qu'on peut comprendre que chaque homme porte en lui un ego transcendantal et comment il peut l'apercevoir par une auto-méditation.

Cette précaution méthodologique implique que la réduction est opérée par moi sur moi ainsi que sur tous les autres ${ }^{33}$, mais qu'elle peut aussi être opérée par autrui de telle sorte qu'il accède à sa subjectivité absolue et me découvre comme alter ego co-fonctionnant avec lui ${ }^{34}$. De la même façon, l'auto-méditation et sa communisation sont opérées en moi et valent alors pour moi et pour les autres en tant que méthode justifiant tout sens, y inclus le sens de la philosophie et celui du devenir authentique de l'humanité. Les autres, également, peuvent opérer cette méthode, ils «peuvent se méditer" (App. XXIV, p. 540). Ajoutons que cette méthodologie détient son efficacité du fait que la réduction et l'auto-méditation ne sont possibles que dans une attitude qui suspend la

32. Pour cette description de la constitution d'autrui et de la réduction à l'intersubjectivité, cf. particulièrement à P.P. 2 , leçons $35,36,47,5.3 ;$ L.F.T. \$ 96 , ainsi quà la $V^{c}$ des Méditations Cartésiennes.

33. "De même que nous pouvons opérer la réduction phénoménologrique sur nos actes propres 1. . I, de même nous pouvons opérer une telle réduction sur les actes d'autrui dont nous prenons conscience par l'intropathie " (P.P.2, leçon 47, p. 189).

34. C. à P.P.2, leçon 54, p. 255 
communication naturelle ${ }^{35}$ pour créer une «solitude philosophique " apte à instaurer une telle méthode de fondation ultime. D'autre part, toute tentative de réduire la réduction phénoménologique conduirait tout simplement à un redoublement de la réflexion de telle sorte que cette réduction de second degré correspondrait à une "itération » de la premiè$\mathrm{re}^{36}$. De la même façon, nous devons supposer qu'une radicalisation de l'auto-méditation phénoménologique ne serait possible que sous la forme d'une réitération.

Ce cadre de la réduction avec ses restrictions fait ressortir principalement que même si, de fait, les autres n'accomplissent pas actuellement la réduction ou l'auto-méditation, la méthode continue de valoir universellement, car son pouvoir de fondation ne renvoie pas à une subjectivité personnelle, mais à la subjectivité transcendantale qui correspond au fonctionnement de la Raison en moi ainsi que dans tous les autres. En principe, cette méthode devrait contraindre quiconque à se rendre à l'autorité de la Raison.

On pourrait, ici, saisir que la réduction radicalisée correspond à l'instauration apriorique d'un accord intersubjectif, par lequel le monde se constitue comme un tout identique avec des apparences différentes pour chacun, qui peuvent être comprises comme des modifications intentionnelles rattachées à la synthèse cohérente et concordante, à laquelle toutes les subjectivités ont accès. Dans l'espace de cette réduction, par le moyen d'échanges et de corrections réciproques opérés dans le même nexus intersubjectif, les subjectivités peuvent accorder progressivement leurs vécus respectifs à un même enchaînement intentionnel pour rétablir toutes les discordances ponctuelles ${ }^{37}$. Cependant, si nous voulons demeurer fidèles aux prescriptions méthodologiques de la réduction, nous devons admettre que les échanges factuels ne se constituent que sur la base et dans l'horizon d'un accord intersubjectif possible se justifiant d'abord dans la méditation intrasubjective. La réalisation d'un accord effectif reste soumise à l'application rigoureuse de

\footnotetext{
35. "La prise de conscience de soi [Selbstbesinnung] que le philosophe commençant a ì accomplir doit l'être non pas dans l'attitude communicative naturelle, mais dans l'attitude pour ainsi dire solipsiste " (P.P.2, leçon 35, p. 82).

36. Cf. P.P.2, leçon 47, p. $192-194$

37. Cf. $\$ 47$; App. XVHI, p. 515.
} 
l'auto-méditation entreprise par un moi humain et qui vaut pour tout autre moi inséré dans cette structure apriorique de la concordance intentionnelle et universelle. Par contre, entre cette concordance anticipée dans le moi qui médite selon la méthode de fondation valable pour tous, et la réalisation d'un accord effectif de tous les moi, qui serait fondé sur le consentement de tous à se diriger selon cette méthode, il y a l'écart d'un idéal à réaliser, l'écart — sinon le "détour " d'une méthode dont le destin se joue entre la visée d'une intention proposée par un homme et son remplissement actif par tous les autres hommes. La méthode de réduction ou d'auto-méditation fonde un accord omnisubjectif dans la sphère intuitive, mais elle n'assure pas que tous s'accordent, dans la sphère de la communication, sur le sens même de la méthode. Cette entente ne serait remplie qu'au moment d'une extension effective, d'un transfert de la méthode d'une subjectivité à tous les autres. Lorsque Husserl affirme que :

La philosophie n'est pas une affaire privée et ne peut se développer en conformité avec son sens propre, selon lequel la véritable méthode a été conquise, que dans la communauté de travail des philosophes et dans un progrès infini, [et que] le devoir de chaque philosophe implique donc aussi qu'il rende possible cette communauté, et donc la possibilité d'adopter lucidement cette méthode (App. XIII, p. 485),

il laisse entendre précisément que l'accomplissement de la tâche philosophique, corrélative à l'établissement et au progrès d'une communauté des philosophes, exige avant tout l'extension de la méthode aux autres avec les mêmes conditions de "lucidité » dans lesquelles cette méthode a été conquise.

Cependant, malgré toutes les précautions de Husserl pour justifier un accord universel dans l'attitude transcendantale, toute l'argumentation visant une méthode de fondation apodictique et démontrant sa valeur universelle se cristallise en une thérie qui doit s'incarner en un discours écrit avec tous les risques d'une objectivation éloignant les lecteurs du sens des prescriptions originelles. Bien que la réduction devait empêcher les mésinterprétations en suspendant le sens objectivé de l'attitude communicationnelle pour accéder à la source originelle du sens et pour retrouver l'assurance d'une expres- 
sion univoque de la Raison, bien qu'elle fonde la possibilité d'une réitération de ce sens original, il n'en demeure pas moins que la réitération de cette méthode présuppose la médiation de sa formulation écrite. La réduction recourt, malgré elle, à l'équivoque du langage communicatif qui est le lieu central d'un accord intersubjectif, mais aussi le moment critique de désaccords perpétuels.

Nonobstant qu'une théorie clairement énoncée puisse être comprise activement par les autres (cf. App. III, p. 410), celle-ci risque pourtant d'être comprise passivement, car on peut hériter du sens des propositions et de la méthode, "alors que n'a pas été hérité le pouvoir de réactivation des archi-commencements et par conséquent des sources de sens pour toute étape ultérieure »(App. III, p. 416). La reprise de la méthode renvoie à une itération de la mise en évidence originelle, mais la méthode elle-même ne peut assurer que sa réitération remplisse parfaitement l'itération originelle. Cette méthode risque également d'être "mécomprise », comme Husserl s'en est plaint si souvent ${ }^{38}$.

Cette compréhension passive et cette mécompréhension peuvent être qualifiées de conditions accessoires qui n'empêchent pas ultimement une réactivation du sens originel de la méthode et qui peuvent relever d'une attitude naïve. Mais, est-ce que nous n'évitons pas alors un obstacle essentiel qui pourrait éprouver radicalement la validité universelle de la méthode, du moins restreindre concrètement sa portée ? Nous avons vu que le «saut » de la méditation égologique à une méditation intersubjective était fondé sur une communisation de la synthèse intentionnelle opérée par la réduction de telle sorte que cette méthode ménageait pour toute subjectivité la possibilité d'une véritable réactivation du sens tel qu'il se déploie originellement. Il ne faut pas oublier que ce saut s'effectue à l'intérieur du discours qui l'énonce et que sa reprise active par les autres nécessite la médiation du discours. Devant la nécessité de cette médiation, la méthode elle-même ne peut plus assurer pour les autres subjectivités une compréhension

38. Cf. particulièrement à l'App. XIII, p. 486 et à là " Postface à mes idées directrices pour une phénoménologie pure ". Trad de L. Kelkel, dans Revue de Métaphysique et de Morale, 62,1957, p. $369-398$. 
symbolique qui s'accorderait parfaitement à la compréhension intuitive pure, obtenue par la méditation égologique.

La méthode de l'auto-méditation assure a priori la communisation immédiate de l'expérience intentionnelle dans l'ego qui l'opère, mais elle n'assure pas que les autres participeront transcendantalement à cette communisation avec la même radicalité. Elle ne peut que le souhaiter. Il n'est peut-être pas nécessaire d' "assurer " cette communisation de la méthode, puisque cette dernière vaut aprioriquement pour toute subjectivité, y inclus toutes celles encore prisonnières de la naïveté. Ce faisant, un doute, une ambiguïté s'inscrivent dans la communication intersubjective que la méthode est incapable de dominer, alors qu'elle devait garantir un accès universel à l'objectivité. Cette équivocité peut toujours être suspendue en anticipant la possibilité d'une recompréhension authentique universelle, on peut toujours supposer que la Raison, amenée à l'expression par une première auto-méditation, viendra à se faire entendre de tous, que tous les hommes en viendront à " tomber d'accord "sur un sens identique ; en attendant cette "entente ", nous pouvons suspecter que les «malentendus», les « désaccords» et tous les «conflits» actuels perturbent indéfiniment une communisation authentique et concrète de la méthode. Ce doute ne justifie ni l'irrationalité ni le scepticisme absolu devant l'éventualité d'une communisation de la méthode ; il devrait, au contraire, nous obliger à enquêter sur les causes et les motifs profonds de ces "désaccords" qui alimentent plus que toute autre chose les divers modes du «douter " que la philosophie tente de lever. La réduction phénoménologique nous conduit précisément au seuil de cette enquête que Husserl a inscrite implicitement dans sa théorie de l'intersubjectivité basée sur une harmonisation des modifications intentionnelles.

Même si Husserl nous invite à ne pas considérer la phénoménologie comme une "littérature », mais plutôt à " acquérir avec elle un regard exercé et méthodique, et, par là seulement, devenir apte à juger par soi-même ${ }^{39}$, nous

39. Edmund Husserl, Philosuphie Première, Ptemière partie, trad. par A.L. Kelkel, Paris, P.U.F., 1970, Appendice, p. 298. 
n'avons tout de même pas le choix de l'aborder autrement qu'en tant que «littérature». Par contre, celle-ci ne prétend pas livrer la Raison comme une autorité définitive, elle appelle plutôt, de manière justifiée, les hommes à se rendre responsables de la Raison, elle appelle à «l'esprit de responsabilité philosophique radicale ${ }^{40}$. Cette responsabilité envers la Raison est peut-être une voie plus assurée vers une philosophie et une humanité authentiques que le recours à une autorité de la Raison. Cependant, par rapport à l'univers politique et pratique des communautés humaines, il faut encore s'interroger sur les causes et les motifs profonds des « conflits» qui peuvent, d'une certaine façon, empêcher l'exercice de cette responsabilité.

\section{B) Reprise de la méthode dans la vie personnelle}

Nos interrogations précédentes soulèvent certaines difficultés au niveau de la réalisation des buts de la philosophie dans le devenir personnel. Avant de les formuler, examinons sommairement le processus de cette réalisation : l'auto-méditation essaie de savoir vers quel but se dirige la volonté dans le contexte de la vie personnelle qu'on désire réaliser authentiquement. Ce but correspond « à se donner à soi-même la forme de l'ego véritable, de l'ego libre, autonome» ( $\$ 73$, p. 301). Le remplissement de ce but sous l'éclairage et l'impulsion de l'auto-méditation s'effectue de la manière suivante: la méditation donne une « représentation anticipatrice [. . . d d'un style du remplissement ultimement univoque de la vie active à venir en général » (App. XXIV, p. 538). Cette anticipation a comme propriété de se transformer nécessairement en "une volonté » qui «domine la vie » de la personne et qui, d'une certaine façon, onde l'être personnel. Cette opération prend l'aspect de linstallation dans son existence d'une «forme du gouvernement de soi-même [Selbstregierung]»(App. XXIV, p. 539), par laquelle la volonté visant tel ou tel but se transforme en un "habitus". L'ensemble du processus consiste à peu près en celui du remplissement d'une intention par l'intermédiaire de la volonté

40. Edmund Husserl, Méditations Cartésiennes, trad. par G. Peiffer et E. Lévinas, Paris, Lib. phil. J. Vrin, $1969, \$ 2$, p. 5. 
qui esquisse, amorce et contrôle son effectuation. La volonté, éclairée par l'auto-méditation, vise une réalisation authentique du devenir personnel ${ }^{41}$. Il est possible que la volonté ne se réalise pas immédiatement, mais alors elle se transforme en " une forme subordonnée de la pulsion, laquelle, si tout se passe bien, si "son temps » arrive [nous soulignons], passe à l'effectivité sous la forme de sa résolution dans un fiat" (App. XXIV, p. 539).

Si l'anticipation du style d'existence ne se transforme pas immédiatement en une effectuation, et si elle prend la forme d'une pulsion soumise aux aléas des conditions favorables pour s'accomplir, alors il est possible que l'intention de ce style d'existence soit déviée. Le phénoménologue est conscient de ce problème : "Ici sont possibles, à cause du manque de clarté du projet gouvernant, des gauchissements, des méprises, bref des errances" (App. XXIV, p. 539). Du même coup, il décèle la faille possible du processus, soit le "manque de clarté ", et propose le moyen approprié pour garder le projet gouvernant dans la clarté : "Tout projet gouvernant, en particulier s'il implique la diversité des médiations, a besoin d'une méditation répétée, répétition au sens d'un renouvellement de l'originalité, de l'évidence de son sens authentique » (App. XXIV, p. 539). Cependant, bien que la méditation maintienne le sens authentique du projet gouvernant, peut-elle s'assurer que ce sens se maintienne chez tous les individus appartenant à la même communauté ou à des communautés différentes, peut-elle s'assurer d'une répétition de la méditation chez tous ces individus, qui puisse instaurer une concordance entre les projets gouvernants, de telle sorte qu'un projet gouvernant authentique se concrétise pour chacun et pout tous? Pour obtenir cette concordance, il faudrait que les autres en général aient adopté l'auto-méditation. Or, cela n'est pas assuré si l'on tient compte du fait que Husserl aperçoit deux couches distinctes dans l'existence humaine: «celle de l'homme qui n'est pas encore une personne au sens plein, dans la mesure où il n'a pas accompli l'ultime auto-méditation et implanté en soi le gouvernement de soi-même, et celle de

41. Nous retrouvons le schéma de ce processus : intention-tolonté [tension|-aciomplissent des buts, escquissé à l'app. XX, p. 521-524, er rapporté au problème de la validarion. 
l'homme qui l'a déjà fait "(App. XXIV, p. 539). Bien sûr, cette concordance est atteinte a priori dans l'auto-méditation, et tout homme peut exercer celle-ci, mais tant que cette méthode ne s'est pas communisée à tous, alors la concordance n'est pas remplie et on ne possède pas encore, à proprement parler, la méthode qui assurerait son remplissement; cette concordance reste une anticipation.

Le fait de ces couches de l'existence personnelle entrave l'effectuation du projet gouvernant dans la mesure où celui-ci se heurte à la volonté de ceux qui n'ont pas encore adopté l'auto-méditation, vivant encore dans l'attitude naïve et dans l'errance. Ces derniers empêchent alors toute communautisation d'un projet gouvernant authentique et peuvent même imposer, à celui qui tente de maintenir le sens authentique de son projet existentiel, un style d'existence en désaccord avec son propre projet et contre sa volonté. Tant qu'un projet gouvernant ne résulte pas d'une «constitution » obtenue par une méditation communisée, les hommes continuent de vivre "dans un régime étatique qui possède une ordonnance de la volonté d'après des relations de domination et de servitude " (App. XXIV, p. 541). Et tant que, dans cette normalité d'existence, la domination qui ordonne la vie des êtres n'est pas elle-même assujettie à la Raison $^{42}$, on ne voit pas encore comment on pourrait parvenir à l'idée d'un "conditionnement extérieur » prenant la forme d'une coopération intersubjective ou d'une coexistence "de sujets agissant transcendantalement les uns sur les autres et, dans une activité commune, constituant comme des cuvres les formations qui sont le bien de la communauté $"{ }^{43}$. Pour que cette coexistence s'établisse, il faut d'abord élucider comment l'auto-méditation peut prendre la forme d'une prise de conscience intersubjective, et il faut rechercher ce qui peut entraver une reprise de l'auto-méditation ${ }^{44}$. À moins d'une

42. Le fait que les philosophes soient " mis au ban de la société " (Annexe III, p. 369) dans le combar qu'ils livrent aux conservateurs de la tradition et qui conduit à des "déchirements" de la culture nationale témoigne que le règne de la domination rationnelle sur la vie sociale n'est pas encore advenu.

43. Edmund Husserl, L.F.T. $\$ 104$, p. 366-367.

44. Même en disant : "le fait d'être constamment orienté à une norme [ . . ] n'habite pas toutes les personnes, il n'est pas complètement développé dans les personnalités de niveau supérieur constitué par les actes intersubjectifs, mais il habite cependant en elles sous la forme de la marche nécessaire du développement et de l'extention d'un esprit de 
reprise continuelle de la méditation, non seulement par le sujet singulier mais par chaque membre de la communauté humaine, et à moins d'éviter toute méprise sur le sens de cette méditation, le projet gouvernant d'un style d'existence authentique risque une errance qui l'écarte sans cesse de la « terre promise».

L'accomplissement des buts énoncés par les ancêtres-philosophes dans le devenir personnel semble être conditionné pur une reprise de l'auto-méditation en tous et chacun. La méditation qui n'assure pas sa propagation universelle perd toute efficacité, du moins dans l'existence concrète de celui qui veut guider sa vie selon un sens rationnel. L'auto-méditation renvoie ainsi à une praxis intersubjective, qui se présente d'abord comme l'idéal d'une communauté des philosophes dont le rayonnement atteint la communauté universelle des hommes. Bien que cette méthode permette la transmission et la reprise de l'idéal d'une coexistence intersubjective, la reprise de la méthode elle-même n'est pas assurée dans le cadre idéal d'une praxis intersubjective. En référant à cet idéal en tant qu'il a déjà pris racine dans l'histoire de l'humanité européenne sous la forme d'une communauté intersubjective des philosophes, Husserl réclame avant tout, pour amener cet idéal à son achèvement, le rétablissement véritable d'une intersubjectivité qui assurerait le maintien, le développement et l'extension d'une communauté de sujets méditant, orientés vers les normes de la Raison pour gouverner leur existence et harmoniser leurs relations interpersonnelles. Dans cette optique, l'abandon de l'auto-méditation responsable, qui a provoqué la crise des sciences européennes, semble correspondre à une rupture de l'intersubjectivité entre l'esprit qui animait les fondateurs de la philosophie et l'esprit de ses héritiers qui ne réaniment plus le sens originel de sa fondation. Cette rupture s'est approfondie par le fait que la correction des buts originels n'est point encore parvenue à une parfaite réciprocité critique, les systèmes philosophiques ayant proliféré sans se rejoindre ( $\$ 56$, p. 222). Husserl croit qu'en réactivant l'attitude radicaliste, en la menant encore plus

normes universellement valables" (Annexe III, p. 355), Husserl ne règle pas pour autant le problème implicite d'une entrave à l'extension de l'esprit de normes qui contribue à différet continuellement et indéfiniment l'adoption de l'auto-méditation au niveau individuel et collectif. 
rigoureusement et en propageant l'idée à l'ensemble des hommes, il serait possible de réparer la rupture dans l'accomplissement de la tâche originelle et, ainsi, de surmonter la crise vécue par les sciences et par l'humanité européennes. Ce faisant, il lance un appel au retour à ce qui a été délaissé, il rappelle à l'humanité de continuer l'accomplissement de la tâche originelle prescrite par les ancêtres, et précisée au cours de la collaboration critique des philosophes. Mais un tel appel à renouer avec l'idéal délaissé ne nous dit rien sur les raisons de son abandon, qui, si elles ne sont pas mises à jour, pourront toujours, à nouveau, causer une rupture dans son accomplissement et même son remisage dans l'Inconscient, pour une période indéfinie, de telle sorte qu'il soit oublié, recouvert, par une couche épaisse de sédiments le rendant inaccessible aux archéologues, ou irrémédiablement défiguré. La seule invocation au renouvellement de l'alliance ne suffit pas à la rétablir effectivement, si l'on n'a pas éclairci les causes profondes de sa. brisure.

Le phénoménologue a bien montré comment les sciences ont dévié du sens originel prescrit par la philosophie, comment elles sont tombées dans le préjugé de l'objectivisme sans qu'aucun savant et aucun philosophe antérieur ne réussissent à surmonter cet obstacle pour atteindre la subjectivité transcendantale, prestatrice de tout sens et de toute validité de sens. Cependant, le questionnement-en-retour sur ce détournement historique de la tâche originelle de la science ne fait que raconter descriptivement l'époque du démembrement d'une entreprise qui se voulait originellement unitaire, avec en arrière-plan ou à l'horizon la nostalgie du retour à la Raison. L'appel au retour à la subjectivité fondatrice et à la méditation phénoménologique n'explique pas les raisons qui ont empêché et qui empêchent ce retour. La seule mise à jour du détournement des sciences de leur tâche originelle, du déplacement de leur sens, n'éclaire pas les raisons profondes de la défiguration et de la perte du sens de la téléologie rationnelle. Le chemin de l'automéditation nous conduit à une impasse, celle d'en appeler à un idéal perdu pour régler ce qui va à l'encontre de cet idéal. Pour Husserl, la modification ou la modalisation du sens de l'idéal philosophique tient surtout à sa 
sédimentation dans le tissu historique, la rupture avec le sens n'est qu'apparente ${ }^{45}$. La méditation, en soumettant les sédiments au tamis critique du questionnement-en-retour, devrait retrouver leur sens vivant, continuellement présent. À l'image de la rupture $d u$ sens et de sa possible bifurcation, Husserl oppose celle de son réveil toujours possible.

$\mathrm{Ne}$ serait-il pas tout aussi légitime d'apercevoir une rupture fondamentale dans l'histoire de cette humanité, qui donne lieu à toutes ces tentatives de fonder une science universelle dirigeant transcendantalement le devenir humain et qui motive, dès lors, le recours à un « idéal » pratiquement inaccessible? N'y a-t-il pas lieu de penser qu'en recourant à l'idéal d'une tâche jamais remplie, jamais parfaitement partagée entre les hommes, on indique implicitement le problème très sérieux d'une brèche non colmatée dans la texture intentionnelle de la vie et de l'histoire de la communauté humaine, empêchant le remplissement et le partage universel de la tâche idéalement prescrite par le Logos de la raison, inscrite sur la pierre fondatrice de l'édifice de l'humanité européenne? L'auto-méditation, pour retrouver et accomplir l'intention originaire de la philosophie dans notre existence, est une solution idéaliste au sens le plus pur du mot, elle appelle une radicalité de la réflexion et de la connaissance de soi qui a toujours été empêcbée par des obstacles non éclaircis.

Entre le projet philosophique aperçu dans l'immanence du sujet méditant et son accomplissement dans la transcendance d'une multiplicité infinie de subjectivités qui participeraient, à partir d'une attitude théorique, à une parfaite coexistence intersubjective dans laquelle la chaine intentionnelle ne se romprait pas, donc entre ces pôles, il y a la lacune ${ }^{46}$

45. Les "expériences négatives dans le détail ne suppriment pas l'universalité de la norme de l'accord, lequel se restaure toujours à nouveau. [. . . I On est d'avance certain que tout doute doit pouvoir être levé et que, à tout non-être correspond (et peut être produit) un être-autrement qui rétablisse l'accord comme à tout néant une réalité effective. En sorte que - et cela joue d'avance un rôle permanent - de la discordance sort la concordance communicative " (App. XVIII, p. 514-515; ff. aussi $\$ 47$, p. $183-187)$.

46. Il est intéressant de noter, en référant à la métaphore de la sédimentation, chère à Husserl, que "lacune », pour la géologie, signifie: " une absence d'une couche de terrain dans une série statigraphique qui révèle une interruprion dans la sédimentation ou une érosion postérieure au dépôt d'une roche » (Petit Larousse). 
d'une communisation de l'auto-méditation, ou encore la suspension d'un accord intersubjectif sur la méthode elle-même. L'approche husserlienne a cependant le mérite d'indiquer le lieu privilégié de la crise du sens dans notre existence. Ce lieu serait celui d'une rupture intersubjective ${ }^{47}$ par rapport aux générations passées et futures et par rapport à la communauté présente des hommes, à l'humanité qui serait pour le phénoménologue "une communauté de pouvoir s'exprimer dans la réciprocité, la normalité et la pleine intelligibilité " (App. III, p. 408). Cette rupture de la chaîne intersubjective se présente principalement dans le problème de l'objectivation du subjectif : elle a lieu autant dans la saisie des personnes comme objets ou co-sujets que dans le fait de rendre intersubjectif ce qui est intrasubjectif par la médiation des objectivités idéales. Que ce soit au niveau de l'intuition intropathique ou de la communication intersubjective, l'objectivation des visées subjectives propres (projets) et leur traduction (accomplissement) dans la transcendance de la vie personnelle, individuelle et communautaire, semblent entraîner des altérations du sens, qui provoquent principalement l'équivoque et l'incertitude. De cette façon, sans définir les possibilités d'une intersubjectivité réelle et d'une compréhension intropathique réciproque entre les hommes, on ne peut véritablement garantir, légitimer, valider l'objectivation du sens. À cet égard, K.O. Apel, qui reprend la tâche d'établir les conditions de la validité du sens, situe les faiblesses de la phénoménologie husserlienne dans sa tentative de fonder le sens sur l'évidence de la conscience subjective, négligeant ainsi la question de sa "validité intersubjective ». Ce qui l'amène à proposer le remplacement de «ce fondement sur l'évidence subjective par le principe régulatif d'une formation critique d'accord dans une communauté idéale de communication qu'on doit tout d'abord instaurer dans la communauté réelle de la communication $\gg 48$.

47. Jürgen Habermas traite de ce problème lorsqu'il parle des "distorsions de la communication " en relation avec sa théorie de la " compétence communicative ». Il nous sembie que ses analyses portent précisément sur les obstacles à la compréhension intersubjective, dont l'idéalisme phénoménologique ne s'est pas tellement préoccupé. Cf. Jürgen Habermas, "On systematically distorted communication " et "Towards a theory of communicative competence " dans Inquirer, 13, 1970, p. 205-218 et p. 360-374.

48. K.O. Apel, Transformation der Philosophie II, Frankfurt am Main, Suhkramp Verlag, 1973, p. 354-355; citation traduite par W. Dallmayr dans "Expérience du sens et 
Il nous semble, en fin de compte, que les autres et le temps sont les principaux obstacles que Husserl rencontre dans sa tentative d'amener la phénoménologie à renouer avec l'idéal de la philosophie ${ }^{49}$, en recourant à l'auto-méditation pour « percer la croûte » de l'histoire $(\$ 7$, p. 24$)$, reprendre le message des ancêtres-philosophes et le faire comprendre à tous les hommes, pour que l'humanité parvienne à se gouverner rationnellement sous l'archontat de la communauté des philosophes ; il y a d'abord le problème de réanimer en soi-même l'intention, les buts des autres, figés dans la texture historique sédimentée ou recouverts par une superposition d'objectivités, il y a ensuite celui de réanimer dans les autres ces buts, cette réanimation impliquant le transfert entre les hommes de l'attitude radicale de l'auto-méditation qui, par ses propres forces et par sa nature, peut seule retrouver le chemin de la réconciliation avec le Logos de la Raison.

Par sa théorie de l'auto-méditation, Husserl renouvelle surtout sa problématisation de la formation du sens. L'automéditation permettrait de mâtriser la formation du sens qui, originairement, vise l'idéal téléologique d'une humanité rationnelle. L'accomplissement effectif de cet idéal dans le devenir de l'humanité dépendrait, alors, d'une radicalisation subjective de la réflexion sous la forme de la méditation. Dans cette perspective, la compréhension imparfaite du sens et son accomplissement différé semblent dépendre d'un manque de radicalité subjective de la réflexion qui ne réussit pas à réactiver la formation originelle et à l'assumer jusqu'à sa pleine réalisation. Cependant, Husserl n'explicite pas comment cette radicalité subjective nous garde d'une déformation du sens telle qu'elle peut se produire effectivement au cours de sa transmission entre les hommes, les communautés et les générations, ou

réflexion sur la validité ; K.O. Apel et la transformation de la philosophie ", dans Aribites de la Philosopbie, 39, 1976, p. 383.

49. «La phénoménologie nous parait tourmentée sinon contestée de l'intérieur par ses propres descriptions du mouvement de la temporalisation et de la constitution de l'intersubjectivité. "

Jacques Derrida, La vioix et le phénomène, P.U.F., Paris, 1976, p. 5.

Jean T. Desanti soutient également qu' "autrui " et le "temps " "font éclater le champ transcendantal " de la phénoménologie.

Jean T. Desanti, Introduction à la phénoménolngie, Coll. Idée, Gallimard, Paris, 1976, p. $132-134$. 
au cours de sa réalisation à travers des institutions sociales. La communisation de l'auto-méditation devient un idéal dont on cherche la méthode efficace pour l'atteindre. L'idéal d'une telle communisation, vers lequel conduit la théorie de l'auto-méditation, nous donne accès, tout de même, à une problématisation transcendantale de la compréhension du sens avec ses horizons dynamiques de transformations et de déformations. Cette problématisation peut contribuer à éclaircir le débat contemporain sur les dimensions épistémologiques et pratiques de la compréhension.

Faculté de Philosophie Université Laval 